\title{
Avaliação funcional em idosas: uma proposta metodológica
}

\section{Functional assessment in elderly: a methodological proposal}

\author{
Cleiton Silva Correa \\ Kelly Cristina de Mello Moraes \\ Fabiano Cézar Lacerda ${ }^{1}$ \\ Regis Radaelli ${ }^{1}$ \\ Anelise Reis Gaya \\ Ronei Silveira Pinto ${ }^{1}$
}

Resumo - O objetivo do presente estudo consistiu em verificar se as alterações no desempenho no teste de salto com contra movimento (CMJ) estão associadas aos incrementos obtidos nos testes para avaliação da capacidade funcional. Para a avaliação da capacidade funcional, realizaram-se os testes 1RM, sentar e levantar e 8 foot up and go, após seis semanas de treinamento de força. A amostra voluntária foi constituída por 59 idosas saudáveis e destreinadas, divididas em grupo controle (GC: $\mathrm{n}=17$ ) e experimental (GE: $\mathrm{n}=42)$. O GE obteve incrementos significativos nos testes CMJ (22,5\%) 1RM (21,5\%), sentar e levantar (22,8\%) e 8 foot up and go (24\%). No GE, incrementos nos valores do CMJ correlacionaram-se significativamente $(\mathrm{p}<0,001)$ com os incrementos nos testes de 1RM $(r=0,88)$, sentar e levantar $(r=0,82)$ e 8 foot up and go $(r=-0,75)$; e apresentaram diferenças significativas $(\mathrm{p}<0,05)$ em relação ao $\mathrm{GC}$ em todas as variáveis. Sendo assim, é possível sugerir a utilização do CMJ como indicador de funcionalidade em mulheres idosas, visto que o teste parece representar adequadamente as alterações funcionais decorrentes de um período de treinamento de força de seis semanas em mulheres idosas.

Palavras-chave: Capacidade Funcional; Força muscular; Idoso.

Abstract - This study aimed to verify whether changes in performance in the countermovement jump test (CMJ) are associated with the improvements observed in the functional capacity tests. Methods: Functional capacity was measured with the IRM, the 30 seconds chair-standing and the 8 foot up and go tests, after six weeks of strength training. The voluntary sample was comprised of 59 healthy untrained elderly women, who were randomly divided into experimental $(E G=42)$ and control groups $(C G=17)$. The $E G$ showed a statistically significant increase in the CMJ (22.5\%), 1RM (21.5\%), 30 seconds chair-standing (22.8\%) and 8 foot up and go (24\%) results. The increases observed in the CMJ test showed a very high significant association $(p<0.001)$ with the increases observed in the functional capacity tests: 1RM test $(r=0.88), 30$ seconds chair-standing test $(r=0.82)$, and 8 foot up and go test $(r=-0.75)$. Such increases were not seen in the CG. Results suggest that CMJ is a good test for measuring functional capacity in elderly women. The CMJ test seems to adequately express the changes in functional capacity observed over a six-week period of strength training. Key words: Elderly; Funcional capacity; Muscle Strength.
1 Universidade Federal do Rio Grande do Sul. Escola de Educação Física. Porto Alegre, RS. Brasil.

Recebido em 10/07/12 Revisado em 24/04/13 Aprovado em 20/06/13

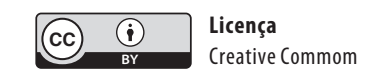




\section{INTRODUÇÃO}

O envelhecimento está associado ao declínio das funções neurais e morfológicas, resultando em decréscimo da força máxima e potência muscular de membros inferiores ${ }^{1}$. Os fatores neurais são responsáveis por alterações no padrão de recrutamento, na taxa de disparo e sincronização das unidades motoras ${ }^{2}$, enquanto os morfológicos são responsáveis pela diminuição da área de secção transversa (AST) e pela atrofia de fibras musculares, principalmente as do tipo II, importantes para a produção de força rápida muscular3.

Dados recentes indicam que o envelhecimento diminui a capacidade de produção de força explosiva mais do que a produção de força máxima ${ }^{3}$. Do ponto de vista funcional, a capacidade de produção de força máxima, em especial, a força explosiva, tem impacto importante nas atividades de vida diária $(\mathrm{AVD})^{4}$, como sentar e levantar de uma cadeira ou subir escadas, que são tarefas dependentes da produção de força muscular ${ }^{2-5}$.

Alguns testes são utilizados para avaliar a capacidade funcional de idosos como o teste de uma repetição máxima (1RM), o teste de sentar e levantar, o foot up and go e testes de salto. O teste de uma repetição máxima (1RM) é capaz de mensurar a força dinâmica máxima. Já, o teste de sentar e levantar verifica o maior número de repetições que o indivíduo consegue concluir ao levantar da cadeira até a posição ereta plena e retornar à posição inicial, concluindo o maior número possível de repetições dentro de um período de 30(s), sendo realizada a contagem verbal (em voz alta $)^{4-6}$.

Outro teste funcional, ofoot up and go, consiste em levantar, deslocar-se em oito passos, contornar o obstáculo e sentar, avaliando o equilíbrio dinâmico e a agilidade de membros inferiores ${ }^{4}$. Também funcionais, os testes de saltos verticais contam com a utilização do ciclo alongamento-encurtamento (CAE) na qual os segmentos do corpo são submetidos a ações excêntricas e concêntricas ${ }^{7}$ durante atividades como correr, saltar e caminhar e avalia a força rápida muscular ${ }^{8,9}$. Um exemplo de teste de salto realizado com uma flexão-extensão rápida de joelhos, com uma parada mínima entre as fases excêntrica e concêntrica, é denominado countermovement jump (CMJ). Este salto é caracterizado como um CAE de longa duração e pode ser usado na medida de força explosiva, na expressão da porcentagem de fibras musculares de contração rápida e na mensuração da utilização da energia elástica.

$\mathrm{O} C \mathrm{CJ}$ é importante para a população deste estudo à medida que, durante a sua execução, como compõe o CAE, parece eficaz para o desenvolvimento da capacidade funcional de mulheres idosas, apresentando papel importante para a realização das $\mathrm{AVD}^{4}$. Além disso, o CMJ exige força dos membros inferiores e a manutenção de uma postura estável, pois a força do quadríceps diminui com o envelhecimento e provoca um declínio do equilíbrio corporal ${ }^{10}$.

Como os exercícios que exigem a ativação do CAE parecem difícil de serem aplicados com idosas, seria interessante realizar estratégias de treinamento de força em que o CAE estivesse inserido ${ }^{9}$ com o objetivo de 
reduzir o tempo de resposta muscular. Neste contexto, a realização de um treinamento de força de membros inferiores torna-se indispensável para possibilitar a realização de AVD, manter a independência funcional, melhorar o equilíbrio corporal e contribuir para a diminuição da incidência de quedas de mulheres idosas ${ }^{10}$.

Apesar da importância do treinamento de força para o desempenho das idosas durante a prática dos testes funcionais, não se sabe se há correlação entre as adaptações decorrentes do treinamento de força, sobretudo, o desempenho do CMJ, com as alterações nos testes de 1RM, sentar e levantar e foot up and go. Essa constatação está de acordo com a perspectiva de que o CMJ poderá ser uma alternativa para a avaliação das AVD, considerando que todas as variáveis que constituem as AVD tenham correlação com esse teste.

Deste modo, a carência de dados na literatura sobre os métodos utilizados para avaliação da capacidade funcional de idosas motivou a elaboração deste trabalho, que se faz importante para demonstrar a importância do treinamento de força e o seu envolvimento nas capacidades funcionais. Nesta perspectiva, apresentamos como hipótese que há correlação entre as adaptações no CMJ e o teste de 1RM, teste de sentar e levantar e teste foot up and go, após um programa de treinamento de força. Sendo assim, o objetivo do presente estudo foi verificar se as alterações no desempenho do teste de salto com contra movimento (CMJ) estão associadas com os resultados obtidos nos testes para avaliação da capacidade funcional: 1RM, sentar elevantar e foot up and go, após seis semanas de treinamento de força.

\section{PROCEDIMENTOS METODOLÓGICOS}

Este trabalho faz parte de um Projeto de Extensão da Universidade Federal do Rio Grande do Sul, financiado pela Capes, com duração de 12 semanas de treinamento de força e 12 semanas de destreino. Este estudo foi realizado com idosas (idade: 65,15 $\pm 4,96$; estatura: $162,06 \pm 6,35$ ) com idade entre 60 e 75 anos da cidade de Porto Alegre-RS. O cálculo amostral foi baseado em estudos ${ }^{11-13}$, sendo que o cálculo foi realizado para amostras emparelhadas através do cálculo do Normograma de Altman, 1982 apud Whitley \& Ball ${ }^{14}$, em que foi adotado um nível de significância de 0,05 , um poder de $80 \%$ e um coeficiente de correlação de 0,7 para todas as variáveis. Com base nos desvios-padrão e nas diferenças entre as médias obtidas dos estudos anteriormente citados, os cálculos realizados demonstraram a necessidade de um "n" de, no mínimo, 30 mulheres para o grupo experimental.

Os sujeitos da pesquisa foram devidamente informados sobre os procedimentos metodológicos do estudo e assinaram o termo de consentimento livre e esclarecido aprovado pelo Comitê de Ética e Pesquisa da Universidade Federal do Rio Grande do Sul (no 19322).

Os critérios de inclusão adotados para a seleção da amostra foram: idosas sem experiência prévia em treinamento de força, sem comprometimento mecânico de membros inferiores, que não apresentassem histórico 
de doenças graves (cardiovasculares, endócrinas, metabólicas e neuromusculares), ou que utilizassem medicamentos com influência no metabolismo endócrino ou neuromuscular.

Para a avaliação da capacidade funcional, foram realizados os seguintes testes: CMJ, 1RM, sentar e levantar e foot up and go.

No teste de countermovement jump (CMJ), o indivíduo foi posicionado de pé próximo à plataforma de força AMTI - (Advanced Mechanical Technology, INC - modelo OR6-WP-1000), com as mãos na linha da cintura e acima das cristas ilíacas, com o intuito de diminuir a participação dos membros superiores durante o salto ${ }^{13}$. Ao comando do controlador do teste, o indivíduo deveria executar um passo para o centro da plataforma de força e realizar um salto o mais vertical possível, buscando atingir o máximo de altura possível sem flexionar os membros inferiores durante a fase de voo. Após a queda, o indivíduo deveria equilibrar-se dentro dos limites da plataforma e ficar na posição estática até o momento da autorização do controlador do teste para sair da posição. Após a compreensão da mecânica do salto, o indivíduo foi instruído a executar vários saltos para dominar a técnica do movimento, aproveitando também como aquecimento específico dos membros inferiores. Posteriormente à familiarização do teste, os sujeitos realizaram três saltos $\mathrm{CMJ}$ na plataforma de força com dois minutos de intervalo entre os saltos. A altura do salto do CMJ foi obtida por meio da plataforma de força e determinada pela equação de Bosco ${ }^{15}$ : [(Altura de salto $=9,8$. $\left.(\text { tempo de voo })^{2} / 6\right)$ ], com a utilização de software Matlab versão 5.3 para a análise dos dados. Ao completarem os três saltos, o salto de maior amplitude foi gravado para posteriores análises ${ }^{16-17}$.

Para avaliar a força máxima de membros inferiores, foi realizado o teste de uma repetição máxima (1RM), no exercício de extensão de joelhos, na "cadeira extensora" da marca WORLD-ESCULPTOR com resolução de $1 \mathrm{~kg}$. Para o controle da velocidade de movimento, foi utilizado um metrônomo da marca QUARTZ com resolução de $1 \mathrm{Hertz}(\mathrm{Hz})$. Após a seleção da carga, cada indivíduo realizou o maior número possível de repetições do exercício. Em cada tentativa, as fases concêntrica e excêntrica tiveram a duração de $2 \mathrm{~s}$, cada uma ${ }^{18}$. Conforme $\mathrm{o}$ número de repetições realizadas, a carga foi redimensionada a partir dos valores propostos por Lombardi ${ }^{19}$, a fim de estimar o valor correspondente a 1RM. O valor de 1RM (Kg) de extensão de joelhos utilizado para as análises caracteriza-se pela maior carga que pode ser suportada em uma repetição de um determinado exercício.

No teste de sentar e levantar, o sujeito deveria sentar em uma cadeira com as costas retas, subir para uma posição ereta plena (corpo ereto e reto) e em seguida, retornar à posição inicial. Cada indivíduo foi incentivado verbalmente a concluir o maior número possível de repetições dentro de um período de $30 \mathrm{~s}^{14}$. Para o controle do tempo, foi utilizado um cronômetro da marca TEKNOS. O número máximo de repetições corretas foi utilizado para posteriores análises. 
O foot up and go teve o propósito de avaliar a agilidade e o equilíbrio dinâmico, que são fatores importantes para tarefas que requerem rápidas ações musculares. O teste foi constituído do número máximo de (s) necessários para se levantar de uma posição sentada, executar oito passos (caminhar em torno de 2.44metros, aproximadamente) e retornar à posição inicial. Os indivíduos tiveram três tentativas, e a tentativa de menor tempo de execução do teste foi utilizada para posteriores análises ${ }^{20}$. Após essa etapa, teve início o processo de intervenção na Escola Superior de Educação Física (ESEF/UFRGS) com a realização do treinamento de força. Para a realização do treinamento de força, os indivíduos foram divididos, por meio de um sorteio, em grupo experimental (GE: $n=42)$ e grupo controle (GC: $\mathrm{n}=17)$.

Nas primeiras três semanas do treinamento, os sujeitos realizaram duas séries de 15 a 20 repetições máximas (RMs) por exercício, e nas semanas seguintes, até a $6^{\mathrm{a}}$ semana, praticaram duas séries de 12 a $15 \mathrm{RMs}$ em cada exercício com 90 a 120s de descanso entre as séries. No início de cada sessão, os indivíduos realizaram um aquecimento em cicloergômetro, durante cinco minutos e após, realizaram os seguintes exercícios: supino reto, puxada frontal, remada alta, leg press, rosca tríceps, extensão de joelho, rosca bíceps e flexão de joelhos. Além disso, cada repetição foi executada lentamente, visando à amplitude completa do movimento, com as fases concêntrica e excêntrica com duração de aproximadamente 2 s cada.

Após o processo de intervenção, os testes de CMJ, 1RM, sentar e levantar e foot up and go foram realizados novamente. Para análise dos dados, foram utilizados o teste de normalidade de Kolmigorov-Smirnov e o teste de homogeneidade de Levene. Para as comparações entre os valores de pré e pós seis semanas de treinamento, foi utilizada a análise de variância (ANOVA) two-way com medidas repetidas (Tempo vs. Grupo) e, para comparar dados percentuais, foram utilizados o teste de post-hoc de Bonferroni e ANOVA oneway. Para verificar as correlações, utilizou-se o teste de correlação de Pearson. O nível de significância $\alpha<0,05$ foi considerado em todas as análises. Para a execução dos procedimentos estatísticos, foi utilizado o pacote estatístico SPSS versão 18.0.

\section{RESULTADOS}

Após as seis semanas de treinamento, o grupo experimental apresentou incrementos significativos $(\Delta \%)$ de: $21,5 \%(21,50 \pm 11,25)$ no CMJ, $22,8 \%$ $(22,78 \pm 11,07)$ no teste de $1 \mathrm{RM}, 24 \%(23,97 \pm 11,23)$ no teste sentar e levantar e $22,5 \%(-22,55 \pm 8,71)$ no foot up and go, comparativamente ao pré-treinamento $(\mathrm{p}<0,05)$ e ao grupo controle $(\mathrm{p}<0,05)$.

O incremento nos valores do CMJ do grupo experimental, após as seis semanas de treinamento, apresentou correlações significativas com o delta percentual $(\Delta \%)$ da variável 1RM (figura 1$)$.

Da mesma maneira, foi verificado um incremento nos valores do CMJ do grupo experimental, após as seis semanas de treinamento, apresentando 
correlações significativas com o delta percentual $(\Delta \%)$ da variável sentar e levantar (figura 2).

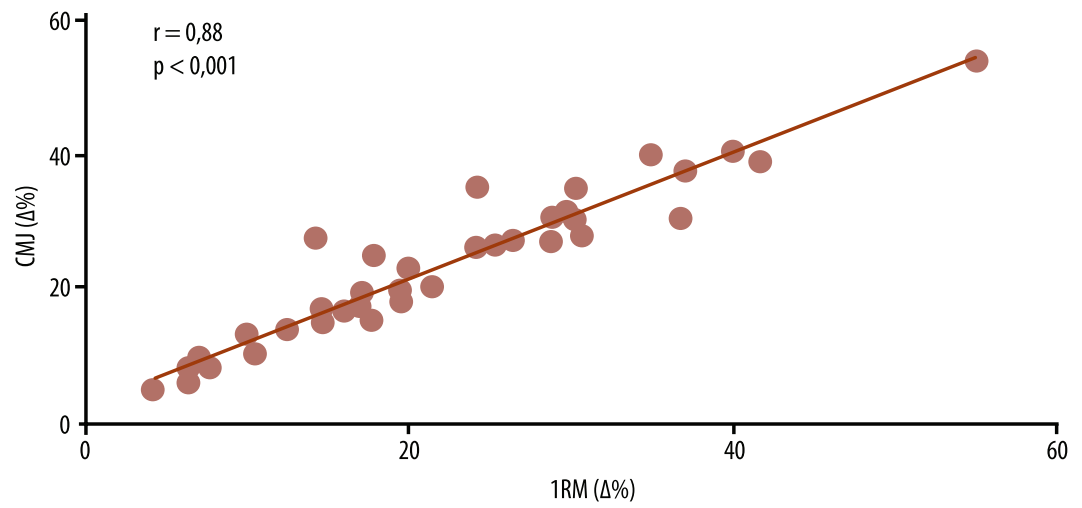

Figura 1. Correlação entre o incremento percentual ( $\Delta \%$ ) dos testes de salto com contra-movimento (CMJ) e 1 RM.

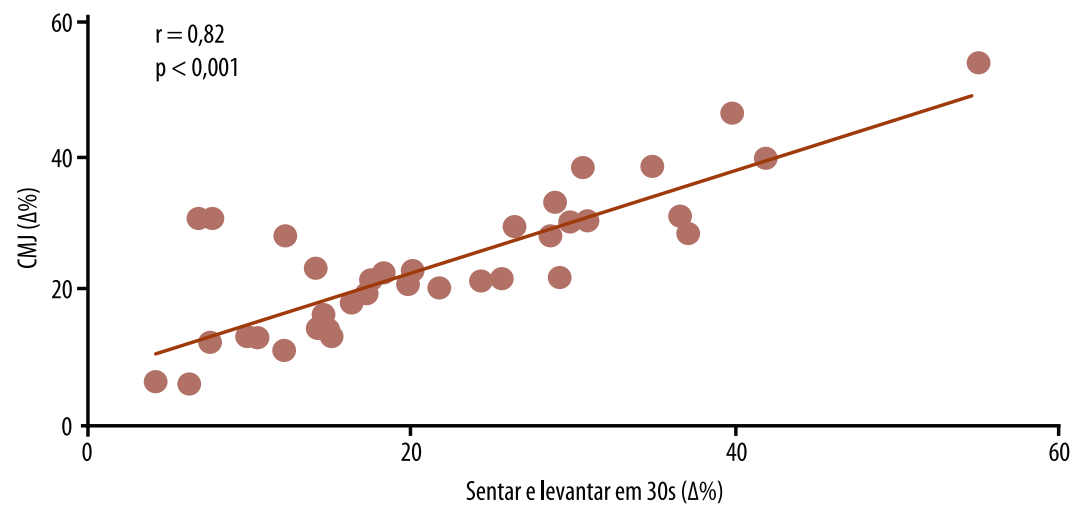

Figura 2. Correlação entre 0 incremento percentual $(\Delta \%)$ dos testes de salto com contra-movimento (CMJ) e Sentar e Levantar em 30s.

Por outro lado, foi verificada uma correlação inversa com o tempo na realização do teste de foot up and go $(\mathrm{r}=-0,75)$ (figura 3$)$.

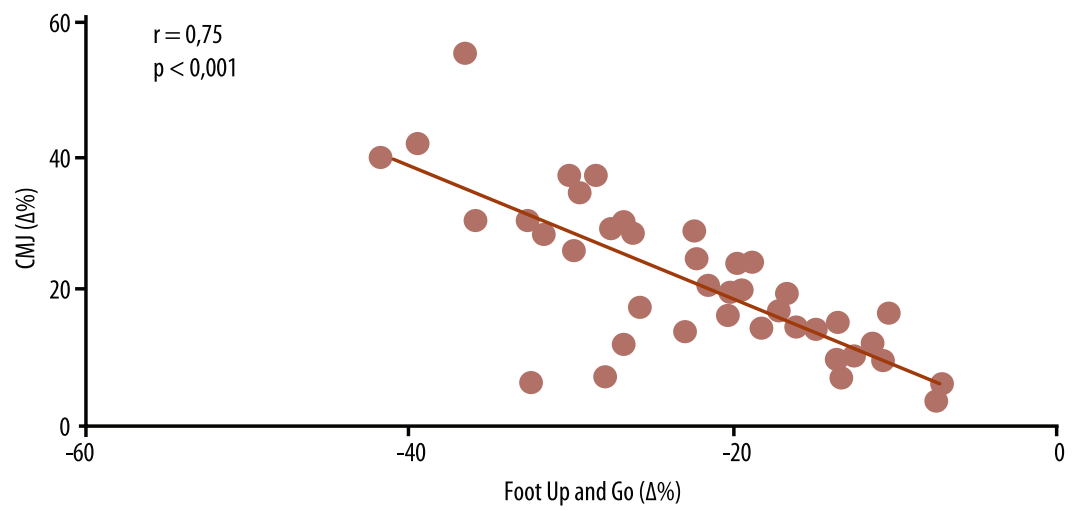

Figura 3. Correlação entre 0 alterações no percentual percentual $(\Delta \%)$ dos testes de salto com contra-movimento (CMJ) e foot up and go.

Para finalizar, o grupo controle não demonstrou alteração em nenhuma das variáveis analisadas após as seis semanas de treinamento. 


\section{DISCUSSÃO}

O objetivo do presente estudo foi analisar a correlação entre as adaptações decorrentes do treinamento de força no desempenho do teste CMJ com as alterações nos testes de 1RM de extensão de joelhos, sentar e levantar e foot up and go, e propor o CMJ como um teste alternativo para a avaliação da capacidade funcional de mulheres idosas. Foram verificadas elevadas correlações entre os valores do desempenho do $\mathrm{CMJ}$ com os deltas percentuais $(\Delta \%)$ de todas as variáveis citadas acima $(r=0,88 ; r=0,82, r=-0,75$, respectivamente).

Esta pesquisa parece justificar-se pela escassez de dados na literatura que comprove a importância do CMJ como um método alternativo para avaliar diferentes manifestações da capacidade funcional, tais como aquelas avaliadas por meio do teste de 1RM, sentar e levantar e foot up and go. Além disso, os resultados do trabalho contribuem para a referida hipótese, sugerindo que a utilização do CMJ parece justificar-se como recurso para a avaliação da capacidade funcional da população de mulheres idosas.

Analisando as correlações em cada teste, em relação à força máxima, primeiramente, os resultados parecem ser explicados pelo fato de que o CMJ e o teste de 1 RM dependem, em grande parte, da força máxima e capacidade de contração dos músculos extensores dos joelhos e avalia a expressão de fibras de contração rápida de membros inferiores ${ }^{7}$.

Da mesma maneira, foi encontrada uma correlação entre as adaptações no CMJ e o teste de sentar e levantar que, provavelmente, devem-se ao fato de ambas as ações serem muito semelhantes em nível articular ${ }^{21}$. Porém, são testes realizados de formas diferentes em que a execução do sentar e levantar apresenta relação com a inércia do movimento (Lei de Newton) por meio da realização de vários movimentos, durante 30s, enquanto, no CMJ, apenas um movimento é realizado.

Adicionalmente, em relação à correlação entre o CMJ e o foot up and go, na perspectiva metodológica, considera-se que ambos os testes utilizam o ATP-CP como substrato energético. O ATP-CP é predominante em atividades musculares intensas e em ações de curta duração ${ }^{22}$.

Além disso, a literatura apóia a ideía de melhoras no CMJ em relação ao $1 \mathrm{RM}^{23}$, sentar e levantar e foot up and go após seis semanas de treinamento de força e revelam que a melhora do desempenho das variáveis analisadas pode estar relacionada a fatores neurais (padrão de recrutamento, taxa de disparo e sincronização das unidades motoras) ${ }^{2}$ e morfológicos (aumento da área de secção transversa e espessura muscular). De fato, nas semanas iniciais de treinamento de força, a ativação neural é predominante em relação aos fatores morfológicos ${ }^{24-27}$.

Nesta perspectiva, os resultados apresentados são importantes por constatarem que o CMJ é um teste de fácil aplicabilidade, barato, realizado em curto espaço de tempo e caracterizado como um método fidedigno das condições de força máxima e rápida do músculo, que apresentam relação com uma adequada execução das AVD. 
Assim, a aplicação do CMJ com idosas brasileiras torna-se relevante por avaliar a capacidade funcional e a sua relação com os movimentos básicos do dia a dia, como caminhar, correr e saltar, por meio de ações concêntricas e excêntricas, proporcionando uma maior geração de força em uma determinada velocidade de encurtamento muscular, durante a transição da fase alongamento-encurtamento?

Portanto, como o CMJ parece essencial para a realização das AVD, assim como para a funcionalidade de mulheres idosas ${ }^{4}$, sua aplicação comprova a hipótese do estudo e corrobora as correlações encontradas, justificando a sua importância para a avaliação da capacidade funcional da população deste estudo.

\section{CONCLUSÕES}

Apesar da importância de propor o CMJ como um importante teste alternativo para a avaliação das capacidades funcionais, o presente estudo foi realizado em uma amostra selecionada voluntariamente o que, por sua vez, impossibilita sugerir o teste de CMJ como método para a avaliação dos parâmetros de capacidade funcional de outras populações. De fato, verificou-se forte correlação entre os testes, contudo, os resultados estão limitados a uma amostra de mulheres idosas.

Assim, quando as populações são diferenciadas, o treinamento pode influenciar as diferentes respostas adaptativas. No entanto, são necessários mais estudos a fim de defender a hipótese aqui sugerida, principalmente, quando se objetiva um estudo da capacidade funcional e seus métodos de avaliação relacionados à população deste estudo. Sendo assim, o presente estudo sugere a utilização do salto CMJ como indicador de alterações funcionais em uma população de mulheres idosas, visto que o CMJ parece representar adequadamente as alterações funcionais decorrentes de um período de treinamento de força de seis semanas em mulheres idosas.

\section{REFERÊNCIAS BIBLIOGRÁFICAS}

1. Hakkinen K, Alen M, Kallinen M. Muscle CSA- force production, and activation of leg extensors during isometric and dinamic actions in middle-aged and older people. J Aging Phys Activ1998;6(3):232-47.

2. Granacher U, Zajner L, Gollhofer G. Strength, power, and postural control in seniors: Considerations for functional adaptations and for fall prevention. Eur J Sport Sci 2008; 8(6):325-40.

3. Rice J, Keogh JWL. Power training: can it improve functional performance in older adults? a systematic review. Int J Exerc Sci 2009;2(2):131-51.

4. Rikli RE, Jones CJ. Development and validation on of a functional fitness test for community- residing older adults. J Aging Phys Activ 1999;7(2):129-61.

5. Macaluso A, De Vito G. Muscle strength, power and adaptations to resistance training in older people. Eur J Appl Physiol 2004;91(4):450-72.

6. Earles D, Judge J, Gunnarsson O. Velocity training induces powerspecific adaptations in highly functioning older adults. Arch Phys Med Rehabil 2000;82:872-878.

7. Komi PV. Stretch-shortening cycle: a powerful model to study normal and fatigued 
muscle. J Biomech 2000;33(10):1197-06.

8. Bosco C, Komi PV. Mechanical characteristics and fiber composition of human leg extensor muscles. Eur J Appl Physiol 1979;41(4):275-84.

9. Cao Z, Maeda A, Shima N, Kuruta H, Nishizono H. The effect of a 12-week combined exercise intervention program on physical performance and gait kinematics in community-dwelling elderly women. J Physiolog Anthropol 2007;3(26):325-32.

10. Shin S, Demura S. The relationship of age and leg strength in the step test with stipulated tempo in the elderly. Arch Gerontol Geriatr 2009;49:311-6.

11. Laroche, D. Initial neuromuscular performance in older women influences response to explosive resistance training. Isokinet Exerc Sci 2009;17:197-205.

12. Caserotti P, Aagaard P, Larsen JB, Puggaard L. Explosive heavy-resistance training in old and very old adults: changes in rapid muscle force, strength and power. Scand J Med Sci Sports 2008;18:773-82.

13. Gerodimos V, Zafeiridis A, Perkos S, Dipla K, Manou V, Kellis S.The contribution of stretch-shortening cycle and arm-swing tovertical jumping performance in children, adolescents, and adult basketball players. Pediatr Exerc Sci 2008;20(4):379-89.

14. Whytley E, Ball J. Statistics review 4: Sample size calculations. Crit Care 2002;6 (4):335-44

15. Bosco C, Luhtanen P, Komi PV. A simple method for measurement of mechanical power in jumping. Eur J Appl Physiol Occup Physiol 1983;50(2):273-82.

16. Caserotti P, Aagaard P, Simonsen EB, Pugaard L. Contraction-specific differences in maximal muscle power during stretch-shortening cycle movements in elderly males and females. Eur J Appl Physiol 2001;84(3):206-12.

17. Souza AL, Shimada SD, Koontz A. Ground reaction forces during the power clean. J Strength Cond Res 2002;16(3):423-27.

18. Spurrs RW, Murphy AJ, Watsford ML. The effect of plyometric training on distance running performance. Eur J Appl Physiol 2003;89(1):1-7.

19. González-Badillo JJ, Sánchez-Medina L. Movement velocity as a measure of loading intensity in resistance training. Int J Sports Med 2007;31(5),347-52.

20. Lombardi VP. Beggining weight training: the safe and effective way. Dubuque 1989.

21. Bottaro M, Machado SN, Nogueira W, Scales R, Veloso J. Effect of high versus low-velocity resistance training on muscular fitness and functional performance in older men. Eur J Appl Physiol 2007;99(3):257-64.

22. Ettema GJC, Huiging PA. Properties of thetendinous structures and series elastic component of EDL muscle-tendon complex of the rat. J Biomech 1989;22(1112):1209-15

23. Costill DL, Coyle EF, Fink WF, Lesmes GR, Witzmann. Adaptations in skeletal muscle following strength training. Eur J Apply Physiol 1979;46(1):96-9.

24. Uchida MC, Bacarau RFP, Navarro F, Pontes FL, Tessuti VD, Moreau RL, et al. Alteração da relação testosterona: cortisol induzida pelo treinamento de força em mulheres. Rev Bras Med Esporte 2004;10(3):165-7.

25. Fiatarone MA, Marks EC, Rian ND, Meredith CN, Lipsitz LA, Evans WJ. High-intensity strength training in nonagenarians: Effects on skeletal muscle. J Am Med Assoc 1990; 263(22):3029-34.

26. Hakkinen K, Kraemer WJ, Newton, RU, Alen, M. Changes in electromyografic activity, muscle fibre and force production characteristics during heavy resistance/ power strength training in middle-aged and older men and women. Acta Physiol Scand 2001;171(1):51-62.

27. Harridge SD, Krieger A, Stensgaard A. Knee extensor strength, activation, and size in very elderly people following strength training. Muscle Nerve 1999;22(7):831-39
Endereço para correspondência

Cleiton Silva Correa

Rua Felizardo, 750 - Bairro: Jardim

Botânico - Lapex

CEP: $90690-200$ - Porto Alegre, RS.

Brasil

E-mail: cleitonesef@yahoo.com.br 